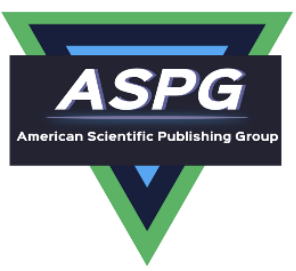

\title{
Using Moving Averages To Pave The Neutrosophic Time Series
}

\author{
Rafif Alhabib ${ }^{1}$, A. A. Salama ${ }^{2}$ \\ ${ }^{1}$ Department of Mathematical Statistics, Faculty of Science, Albaath University, Homs, Syria; \\ rafif.alhabib85@gmail.com \\ ${ }^{2}$ Department of Mathematics and Computer Science, Faculty of Science, Port Said University, Port Said, Egypt; \\ drsalama44@gmail.com
}

\begin{abstract}
In this paper, were using moving averages to pave the Neutrosophic time series. similar to use moving averages to pave the classical time series . the difference, here were dealing with inaccurate data and values of the time series.in the Neutrosophic time series, each unit of time(t) corresponds to a range of values instead of a single value. Finally, we find that the Neutrosophic time series provide an accurate description of the behavior of the series better than in the classic. Therefore, can predict the future of the series as accurately as possible.
\end{abstract}

Keywords: Time Series, Neutrosophic logic, Neutrosophic Time Series, moving averages.

\section{Introduction}

A time series is a set of data arranged in chronological order, the data of this series are associate to each other in the general case, and this correlation gives us reliable future forecasts. also define it as a set of consecutive values (observations) that describe the evolution of a phenomenon over time. say about this time series that it is neutrosophic time series (NTS), if some or all of its values (its observations) are not explicitly specific, such as being a range of values instead of one value [9]. That is, successive observations "that describe the evolution of a phenomenon with time", some or all of it is not precisely defined.

The neutrosophic logic was established by F. Smarandache in 1995 . It is a new branch of philosophy, presented as a generalization for the fuzzy logic [1] and as a generalization for the intuitionistic fuzzy logic [3]. Where presented it as a type of formal logic that aims at explaining the truth, falsehood, and neutral propositions .

The fundamental concepts of neutrosophic set, introduced by Smarandache in $[4,5,6,8]$, Salama and rafif et al. in $[7,9,10,11,12,13,14,15,16,17]$, provides a new foundation for dealing with issues that have indeterminate data. The idea of basic neutrosophic statistics was developed by F. Smarandache [2]. The idea of inferential neutrosophic statistics and neutrosophic statistics was developed by Aslam [18,19]. Singh and Hong presented the time series dataset in to Neutrosophic series using three different memberships are truth-membership, indeterminacymembership and falsity-membership[23]. In this paper, the authors provide a method for paving the Neutrosophic time series using "forward, backward and central" moving averages. This is done similarly to that in classical logic, with the difference that dealing with a Neutrosophic series rather than a classic series.

The aim of this paper is to demonstrate the method of "paving time series using moving averages" within the framework of the Neutrosophic logic. Where find that dealing with the Neutrosophic series is better than the classic 
series, because the Neutrosophic series provides a more accurate and comprehensive description of the time series data set and thus describe the series behavior better than it is in the classic. Which provides a better environment and base for future forecasting.

Moving averages mean dividing the series into a number of equal and overlapping divisions, and replacing each section with a number (mean, median, or others).

\section{The forward moving averages of a Neutosophic time series:}

Symbolize the moving averages of a Neutrosophic time series with the symbol $\mathrm{Nm}_{t}$.

If denote to the Neutrosophic time series with the symbol $N Q_{t}$, the forward moving average is given by:

$N m_{t}=\frac{1}{n}\left[\sum_{i=t}^{t+n-1} N Q_{i}\right]$

Where $\mathrm{n}$ is the degree of the moving average.

For example, for $n=3$ we have:

$N m_{t}=\frac{1}{3}\left[\sum_{i=t}^{t+2} N Q_{i}\right]=\frac{1}{3}\left[N Q_{t}+N Q_{t+1}+N Q_{t+2}\right]$

Therefore:

$N m_{1}=\frac{1}{3}\left[N Q_{1}+N Q_{2}+N Q_{3}\right]$

$N m_{2}=\frac{1}{3}\left[N Q_{2}+N Q_{3}+N Q_{4}\right]$

And so on..

\subsection{Example:}

Let's calculate the forward triple moving average for the following Neutrosophical time series, a series that represents the humidity recorded during seven days in Homs:

\begin{tabular}{|c|c|l|}
\hline$t$ & $N Q_{t}$ & \multicolumn{1}{|c|}{$N m_{t}$} \\
\hline 1 & {$[50,52]$} & $=\frac{1}{3}[[50,52]+[51,53]+[49,50]]=[50,51.67]$ \\
\hline 2 & {$[51,53]$} & $=\frac{1}{3}[[51,53]+[49,50]+[55,58]]=[51.67,53.67]$ \\
\hline 3 & {$[49,50]$} & $=[54.67,56.67]$ \\
\hline 4 & {$[55,58]$} & $=[58,61]$ \\
\hline 5 & {$[60,62]$} & $=[61,64]$ \\
\hline 6 & {$[59,63]$} & \\
\hline 7 & {$[64,67]$} & \\
\hline
\end{tabular}

Table (1): Calculating the forward moving average 


\section{Backward Moving Averages of a Neutrosophic Time Series:}

The Backward moving average for a Neutrosophic time series is given by the following relationship:

$$
N m_{t}=\frac{1}{n}\left[\sum_{i=t}^{t-n+1} N Q_{i}\right]
$$

Where $\mathrm{n}$ is the degree of the moving average.

For example, for $\mathrm{n}=3$ we have:

$$
N m_{t}=\frac{1}{3}\left[\sum_{i=t}^{t-2} N Q_{i}\right]=\frac{1}{3}\left[N Q_{t}+N Q_{t-1}+N Q_{t-2}\right]
$$

Therefore:

$N m_{3}=\frac{1}{3}\left[N Q_{3}+N Q_{2}+N Q_{1}\right]$

$N m_{4}=\frac{1}{3}\left[N Q_{4}+N Q_{3}+N Q_{2}\right]$

And so on..

\subsection{Example:}

Let's calculate the Backward triple moving average for the Neutrosophical time series in the previous example (2.1):

\begin{tabular}{|c|c|l|}
\hline$t$ & $N Q_{t}$ & \multicolumn{1}{|c|}{$N m_{t}$} \\
\hline 1 & {$[50,52]$} & \\
\hline 2 & {$[51,53]$} & \\
\hline 3 & {$[49,50]$} & $=\frac{1}{3}[[50,52]+[51,53]+[49,50]]=[50,51.67]$ \\
\hline 4 & {$[55,58]$} & $=\frac{1}{3}[[51,53]+[49,50]+[55,58]]=[51.67,53.67]$ \\
\hline 5 & {$[60,62]$} & $=[54.67,56.67]$ \\
\hline 6 & {$[59,63]$} & $=[58,61]$ \\
\hline 7 & {$[64,67]$} & $=[61,64]$ \\
\hline
\end{tabular}

Table 2: Calculating the Backward moving average

\section{Central moving average of a Neutrosophic time series:}

The central moving average of a Neutrosophic time series is given by the following relationship:

$N m_{t}=\frac{1}{n}\left[\sum_{i=t-(n-1) / 2}^{t+(n-1) / 2} N Q_{i}\right]$

Where $\mathrm{n}$ is the degree of the moving average.

For example, for $\mathrm{n}=3$ we have: 
$N m_{t}=\frac{1}{3}\left[\sum_{i=t-1}^{t+1} N Q_{i}\right]=\frac{1}{3}\left[N Q_{t-1}+N Q_{t}+N Q_{t+1}\right]$

Therefore:

$N m_{2}=\frac{1}{3}\left[N Q_{1}+N Q_{2}+N Q_{3}\right]$

$N m_{3}=\frac{1}{3}\left[N Q_{2}+N Q_{3}+N Q_{4}\right]$

For example, for $\mathrm{n}=5$ we have:

$N m_{t}=\frac{1}{5}\left[\sum_{i=t-2}^{t+2} N Q_{i}\right]$

And so on..

\subsection{Example:}

Let's calculate the central triple moving average for the Neutrosophical time series in the previous example (2.1):

\begin{tabular}{|c|c|l|}
\hline$t$ & $N Q_{t}$ & \multicolumn{1}{|c|}{$N m_{t}$} \\
\hline 1 & {$[50,52]$} & \\
\hline 2 & {$[51,53]$} & $=[50,51.67]$ \\
\hline 3 & {$[49,50]$} & $=[51.67,53.67]$ \\
\hline 4 & {$[55,58]$} & $=[54.67,56.67]$ \\
\hline 5 & {$[60,62]$} & $=[58,61]$ \\
\hline 6 & {$[59,63]$} & $=[61,64]$ \\
\hline 7 & {$[64,67]$} & \\
\hline
\end{tabular}

Table 3: Calculating the central moving average

\section{Note:}

Through the previous three tables (1),(2),(3) we find that:

- When using the forward moving average, lose from the end of the series a number of values equal to (n-1) value, (in our example the degree of Moving average $n=3$, so lost two values from the end of the series).

- When using the backward moving average, lose from the beginning of the series a number of values equal to (n-1) value, (in our example the degree of Moving average $n=3$, so lost two values from the beginning of the series). 
- When using the central moving average, lose from the beginning of the series and at the end of it a number of values equal to (n-1) $/ 2$ value, (in our example the degree of moving average $n=3$, therefore lost a value from the beginning of the series and a value from the end of the series).

\section{Graphical representation of the time series:}

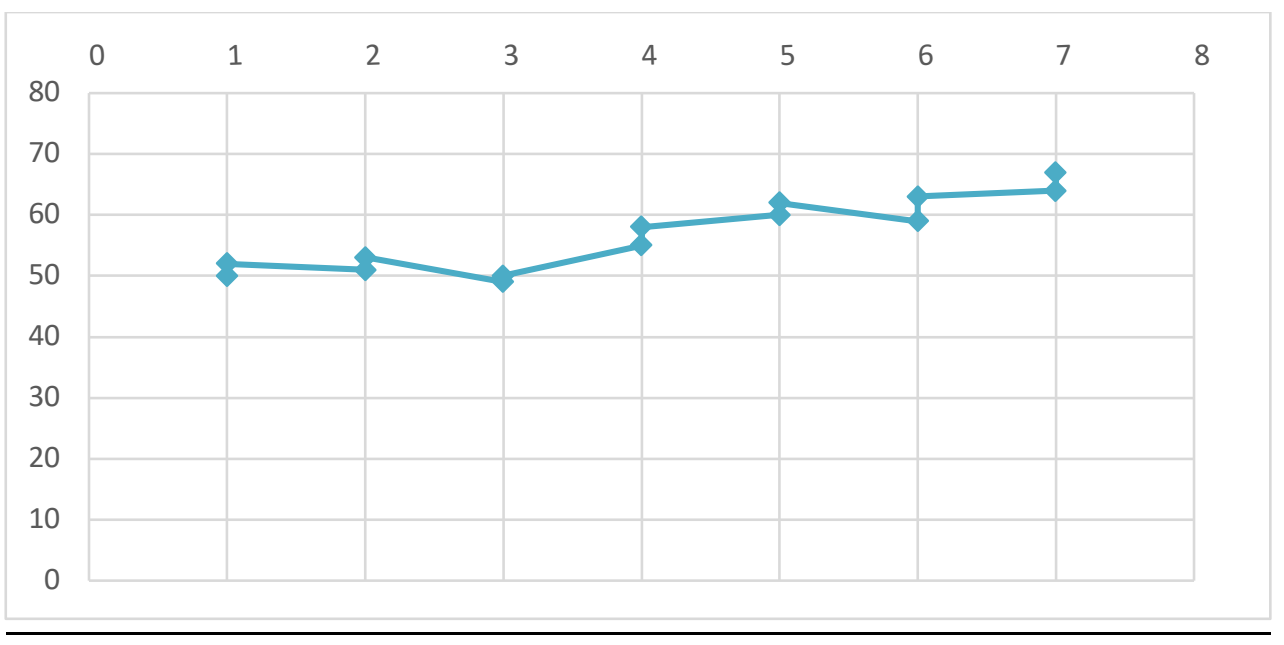

Figure 1. Before paving

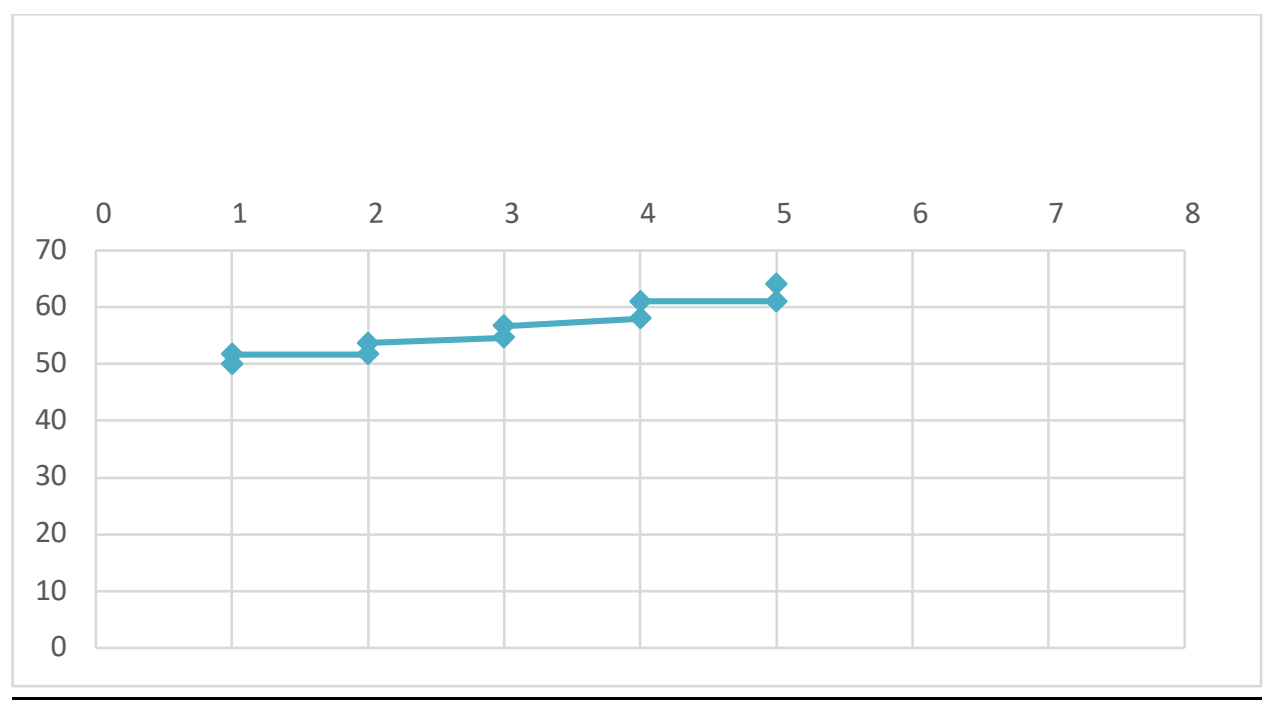

Figure 2. After paving

- conclude from Figures (1) and (2): That the graph line in the series after paving has become smoother than the graph line in series before paving . 
- The diagram of the series did not merge "before and after the paving " into a single diagram until the Neutrosophical values appear clearly and do not cause any confusion.

- Was used the forward moving average in the graph.

\section{Conclusion}

conclude that the use of the Neutrosophic time series and paving them using the moving averages method provides us with a complete and accurate description of the behavior of the time series, which facilitates the prediction process for the future of this series, as well as the prediction process is performed accurately and with the least possible errors.

In the near future, we are looking forward to studying the seasonal, periodic and random changes of neutrosophic time series, as well as the method of eliminating the seasonal effect of neutrosophic time series.

\section{REFERENCES}

[1] ZADEH, L. A." Fuzzy Sets," Inform. Control 8,1965.

[2] Smarandache, F." Introduction to Neutrosophic statistics," Sitech \& Education Publishing, 2014.

[3] Atanassov .k, "Intuitionistic fuzzy sets," In V. Sgurev, ed., ITKRS Session, Sofia, June 1983, Central Sci. and Techn. Library, Bulg. Academy of Sciences, 1984.

[4] Smarandache, F, "Neutrosophy and Neutrosophic Logic," First International Conference on Neutrosophy , Neutrosophic Logic, Set, Probability, and Statistics University of New Mexico, Gallup, NM 87301, USA,2002.

[5] Smarandache, F. "A Unifying Field in Logics: Neutrosophic Logic," Neutrosophy, Neutrosophic Set, Neutrosophic Probability. American Research Press, Rehoboth, NM, 1999.

[6] Smarandache, F, "Neutrosophic set a generalization of the intuitionistic fuzzy sets," Inter. J. Pure Appl. Math., 24, pp.287 - 297, 2005.

[7] Salama, A. A, Smarandache, F, and Kroumov, V, “Neutrosophic crisp Sets \& Neutrosophic crisp Topological Spaces. Sets and Systems," 2(1), pp.25-30, 2014.

[8] Smarandache, F. \& Pramanik, S. (Eds). (2016). New trends in neutrosophic theory and applications. Brussels: Pons Editions.

[9] Alhabib.R, The Neutrosophic Time Series, the Study of Its Linear Model, and test Significance of Its Coefficients. Albaath University Journal, Vol.42, 2020. (Arabic version).

[10] Alhabib.R, Ranna.M, Farah.H and Salama, A. A, "Neutrosophic Exponential Distribution," Albaath University Journal, Vol.40, 2018. (Arabic version).

[11] Alhabib.R, Ranna.M, Farah.H and Salama, A. A, "studying the random variables according to Neutrosophic logic," Albaath- University Journal, Vol (39), 2017. (Arabic version).

[12] Alhabib.R, Ranna.M, Farah.H and Salama, A. A, Neutrosophic decision-making \& neutrosophic decision tree. Albaath- University Journal, Vol (40), 2018. (Arabic version).

[13] Alhabib.R, Ranna.M, Farah.H and Salama, A. A, "Studying the Hypergeometric probability distribution according to neutrosophic logic,"Albaath- University Journal, Vol (40), 2018.(Arabic version).

[14] Salama, A. A., Smarandache, F. “Neutrosophic Crisp Set Theory," Educational. Education Publishing 1313 Chesapeake, Avenue, Columbus, Ohio 43212, 2015.

[15] Salama, A. A. and Smarandache, F. "Neutrosophic crisp probability theory \& decision making process." Critical Review: A Publication of Society for Mathematics of Uncertainty, vol. 12, p. 34-48, 2016. 
[16] Alhabib, R., M. Ranna, H. Farah and Salama, A. A, "Foundation of Neutrosophic Crisp Probability Theory", Neutrosophic Operational Research, Volume III , Edited by Florentin Smarandache, Mohamed Abdel-Basset and Dr. Victor Chang (Editors), pp.49-60, 2017.

[17] Alhabib, R., Ranna, M., Farah, H. and Salama, A. “ Some neutrosophic probability distributions," Neutrosophic Sets and Systems, 22, pp.30-38, 2018.

[18] Aslam, M., Khan, N. and Khan, M.A. "Monitoring the Variability in the Process Using the Neutrosophic Statistical Interval Method," Symmetry, 10 (11), 562, 2018.

[19] Aslam, M., Khan, N. and AL-Marshadi, A. H,"Design of Variable Sampling Plan for Pareto Distribution Using Neutrosophic Statistical Interval Method," Symmetry, 11 (1), 80, 2019

[20] Aslam, M. "Control Chart for Variance using Repetitive Sampling under Neutrosophic Statistical Interval System" IEEE Access, 7 (1), pp.25253-25262, 2019.

[21] Victor Christianto , Robert N. Boyd , Florentin Smarandache, "Three possible applications of Neutrosophic Logic in Fundamental and Applied Sciences," International Journal of Neutrosophic Science, Volume 1, Issue 2, PP: 90-95 , 2020.

[22] Madeleine Al- Tahan, "Some Results on Single Valued Neutrosophic (Weak) Polygroups," International Journal of Neutrosophic Science, Volume 2 , Issue 1, PP: 38-46, 2020.

[23] Singh, P. and Huang, Y.-P." A New Hybrid Time Series Forecasting Model Based on the Neutrosophic Set and Quantum Optimization," Computers in Industry (Elsevier), 111, pp.121-139, 2019. 